\title{
Super-Elastic Double-Helix Model of Photon. Huygens-de Broglie Particle on the Helical Path Guided by the Newton-Bohm Entangled Helical Evolute. Quantum of Magnetic Flux Based on the Mathematical Beauty of Newton, Lorentz, Einstein, Dirac, Gell-Mann, Schwinger, Polchinski and Witten (16.06.2019)
}

\author{
Jiří Stávek ${ }^{1}$ \\ ${ }^{1}$ Bazovského, Prague, Czech Republic \\ Correspondence: Jiří Stávek, Bazovského 1228, 16300 Prague, Czech Republic. E-mail: stavek.jiri@seznam.cz \\ Received: June 15, 2019 \\ Accepted: July 1, 2019 \\ Online Published: July 15, 2019 \\ doi:10.5539/apr.v $11 \mathrm{n} 4 \mathrm{p} 40$ \\ URL: http://dx.doi.org/10.5539/apr.v11n4p40
}

\begin{abstract}
In our approach we have combined knowledge of Old Masters (working in this field before the year 1905), New Masters (working in this field after the year 1905) and Dissidents under the guidance of Louis de Broglie and David Bohm. In our model the photon is represented as the Huygens-de Broglie's particle on the helical path (full wave) guided by the Newton-Bohm entangled evolute (empty wave). We have formulated the concept of the Super-Elastic Photon WAVE based on the Great Works of Weber, Abbe, Voigt and Einstein. This model works with the longitudinal elasticity of that WAVE that was already very well tested experimentally. Newly, we propose to test the elastic amplitude of this WAVE for the case of the Doppler's redshift, the Doppler's blueshift, and the Zwicky's redshift. We have newly used the concept of the Lorentz' force for the description of the photon acting force and the fermion reacting force. In this model the Lorentz' factors $\gamma$ and $\gamma^{3}$ do not describe the "transverse mass of fermions" and longitudinal mass of fermions" but the "reacting transverse force of fermions" and the "reacting longitudinal force of fermions". (The mass of photons and fermions does not change with their speed). It is very well-known that the cylindrical helix observed from different angles forms shadows in the Plato's Cave as circle, sine, cosine, trochoid, cochleoid, hyperbolic spiral. Therefore, the resulting shape depends on the observer position in the Plato's Cave-this is the famous Rashomon effect between observers. Based on the Newton-Bohm helical evolute and the Huygens-de Broglie helical path of the particle we have derived interesting formula known as the quantum of the magnetic flux. When we work further with this concept based on the Mathematical Beauty developed by Dirac, Gell-Mann, Schwinger, Polchinski, Witten and many others, we will obtain possible properties of the magnetic monopole. This photon quantum of the magnetic flux can be experimentally evaluated in the known tests with superconductors and micro-WAVES and infrared-WAVES. Can it be that Nature cleverly works with the magnetic monopole hidden in plain sight? We want to pass this concept into the hands of Readers of this Journal better educated in the Mathematics and Physics.
\end{abstract}

Keywords: Huygens-De Broglie Particle on the Helical Path, Newton-Bohm Entangled Helical Evolute, Super-Elastic Photon WAVE (Weber, Abbe, Voigt, Einstein), Elastic Amplitude of the Photon WAVE, Lorentz' Transverse and Longitudinal Force, Shadows in the Plato's Cave, Rashomon Effect, Born Rule, Heisenberg Relation, Quantum of Magnetic Flux, Magnetic Monopole, "Nature Loves to Hide", Hidden in Plain Sight

\section{Introduction}

The famous quote of Heraclitus "Nature loves to hide" was described in details by Pierre Hadot in 2008. Hadot in his valuable book gives us many examples how Nature protects Her Secrets. In several situations the enormous research of many generations is strongly needed before the right "recipe" unlocking the True reality can be found. Johann Wolfgang Goethe remarked to our research: "Nature does not suffer Her veil to be taken from Her, and what She does not choose to reveal to the spirit, thou wilt wrest from Her by levers and screws." In our model of the photon we have found that Nature could keep Her Secrets hidden in plain sight and thus can perfectly document Her Top Art of Hiding.

Cylindrical Helix represents one of the most fascinating curves in Nature and Science and is among the oldest 
curves. Cylindrical Helix belongs to the Treasure of Geometry. The Cylindrical Helix seem to have been discovered and thoroughly studied by Apollonius of Perga (The Great Geometer) and his scholars as e.g. Geminus of Rhodes. Cylindrical helix is composed from two motions-circular and translational. Heron discovered the cylindrical helix construction by triangle wrapping that gives to us a deeper view in the properties of the elastic helical WAVE. For the details of the Heron's construction see P. Mancosu and A. Arana (2010).

Helices can be observed in many natural forms and structures-see e.g. T.A. Cook in "The Curves of Life" (1914). One of the most fascinating natural structures is the DNA double-helix discovered by two Great New Masters James D. Watson and Francis H. Crick. See e.g., J.D.Watson in 2001 and G. Williams in 2019.

The model of the double helix for the description of the photon wave appeared many times in the so-called dissident literature (outside of the mainstream literature). There are known many proposals for this double helix composition. Louis de Broglie proposed at the 1927 Solvay Conference his model of the full wave and the pilot guiding wave but could not give a deeper physical interpretation of his concept. Later Louis de Broglie (1939) proposed two component model of the photon. (Many modern Dissidents continue to develop this double-helix model of the photon where both helical paths are occupied by particles). In 1952 David Bohm rediscovered this pilot wave model and developed it as the de Broglie-Bohm theory. Since that time the concept of empty guiding waves remains still open and has been waiting for the physical interpretation. See J.S. Bell in 1992, L. Hardy in 1992, P.J. Lewis in 2007, W. Seager in 2018, and many others.

Albert Einstein called these empty waves in the de Broglie-Bohm theory as the ghost waves ("Gespensterfelder", ghost fields) and the de Broglie-Bohmian concept as "too cheap". Are we able to find a physical meaning for those empty waves? In such case we might express here the old Bohemian proverb "Salt above Gold" as "Photons above Gold".

In order to achieve our target we have combined knowledge of Old Masters (working in this field before the year 1905), New Masters (working in this field after the year 1905), and Dissidents working on the double helix model of the photon for many years.

(We are aware of the famous quote of Richard Feynman from the year 1965: "I think I can safely say that nobody understands quantum mechanics.")

\section{Inspirations from Old Masters, New Masters, and Dissidents}

Table 1. Inspirations from Great Masters to formulate a model for photon

FULL WAVE: Huygens-de Broglie particle on the helical path

EMPTY WAVE: Newton-Bohm entangled guiding helical evolute

H.A. Lorentz acting force of photons and reacting force of fermions

Super-elasticity of photon WAVES-Doppler's super-elasticity, Zwicky's super-elasticity

Dirac's quantum of magnetic flux where $\alpha$ is the helical angle

$\Theta_{D}=\frac{h}{e}=\frac{h}{e}(\cos \alpha)^{2}+\frac{h}{e}(\sin \alpha)^{2}$

Superconducting magnetic flux for $\alpha=45^{\circ}$

$\Theta_{0}=\frac{h}{2 e}$

Model of quarks by Gell- Mann and Zweig

Models of dyons by Schwinger

Model of dyons by Witten

$\frac{e \theta}{2 \pi} \quad$ for $\theta= \pm \pi \quad \pm \frac{e}{2}$

Schwinger-Zwanziger condition

$e_{1} g_{2}-e_{2} g_{1}=n h$

$\frac{e}{2} \frac{h}{e}-\left(-\frac{e}{2}\right) \frac{h}{e}=h$

Polchinski: , The existence of monopoles is one of the safest bets that one can make about physics not yet seen“.

We were inspired by many Great Researchers working in this field for generations. Some of those Masters with their ideas are given in the Table 1. 


\section{Heron's Cylindrical Helix Construction by Triangle Wrapping}

Heron of Alexandria invented an elegant construction of the cylindrical helix by triangle wrapping. For the cylinder with radius $\lambda_{0} / 2 \pi$ and the helix pitch $\lambda_{0}$ we will get the starting geometry of the cylindrical helix useful for the modelling of the Super-elastic WAVE. This Heron's Triangle appears frequently in modern Dissident papers, e.g. in R.A. Ashworth in 1998.

In the acronym WAVE the following names of the Great Masters are hidden:

Weber Wilhelm Eduard who derived for the helical path the expression of the light speed as $\mathrm{c}_{\mathrm{W}}=\sqrt{2} *_{\mathrm{c}}$ in 1855 (see e.g. A.K.T. Assis in 2003, F.D. Tombe in 2019),

Abbe Ernst Karl who studied the diffraction of light and derived his famous formula in 1873,

Voigt Woldemar who studied the elasticity of photon waves in the transverse direction in 1887.

Einstein Albert who studied the elasticity of the spacetime in the longitudinal direction in 1905.

The Heron's Triangle will be later modified by the Doppler's redshift, the Doppler's blueshift, and the Zwicky's redshift. The Super-elastic WAVE will give the identical results in the longitudinal direction as for the case of the elastic spacetime theory. Newly, we propose to measure the elasticity of photon waves in the transverse direction using a method employed by R.A. Ashworth in 1998.

\section{Doppler's Longitudinal Redshift of the Super-Elastic WAVE}

For this case when the source and observer are moving away from each other the photon longitudinal wavelength $\lambda_{0}$ will be redshifted by the Lorentz factor $\gamma$ while the cylindrical circumference will be shorted by the factor $\gamma^{-1}$. The frequency $v_{0}$ of the particle in the longitudinal and transverse direction will be smaller by the factor $\gamma^{-1}$. The longitudinal light velocity will be $\mathrm{c}$ while the transverse light velocity will be $\mathrm{c}^{*} \gamma^{-2}$. We propose to measure the amplitude of that Super-Elastic WAVE.

In the Table 2: index $\mathrm{L}$ is for the longitudinal direction, index $\mathrm{T}$ is for the transverse direction, $\mathrm{A}$ is the amplitude and $\mathrm{O}$ is the circumference, $\lambda$ is the wavelength, $v$ is the frequency, $\mathrm{T}$ is the period, $\mathrm{c}$ is the light speed, $\gamma$ is the Lorentz factor.

Table 2. Doppler's Longitudinal Redshift of the Super-Elastic WAVE

$$
\begin{aligned}
& \lambda_{L}=\lambda_{0}\left(1+\frac{v}{c}\right) \gamma=\lambda_{0} \frac{\sqrt{1+\frac{v}{c}}}{\sqrt{1-\frac{v}{c}}} \\
& \nu_{L}=\nu_{T}=\frac{\nu_{0}}{1+\frac{v}{c}} \gamma^{-1}=\nu_{0} \frac{\sqrt{1-\frac{v}{c}}}{\sqrt{1+\frac{v}{c}}} \\
& c_{L}=c \\
& T_{L}=T_{T}=T_{0} \gamma \\
& A=\frac{\lambda_{0}}{2 \pi} \gamma^{-1} \\
& O=2 \pi \frac{\lambda_{0}}{2 \pi} \gamma^{-1} \\
& c_{T}=c \gamma^{-2}
\end{aligned}
$$

\section{Doppler's Longitudinal Blueshift of the Super-Elastic WAVE}

For this case when the source and observer are moving towards each other the photon longitudinal wavelength $\lambda_{0}$ will be blueshifted $\gamma^{-1}$ by the Lorentz factor while the cylindrical circumference will be bigger by the factor $\gamma$. The frequency $v_{0}$ of the particle in the longitudinal and transverse direction will be higher by the factor $\gamma$. The longitudinal light velocity will be $\mathrm{c}$ while the transverse light velocity will be $\mathrm{c}^{*} \gamma^{2}$. We propose to measure the 
amplitude of that Super-Elastic WAVE.

In the Table 3: index $\mathrm{L}$ is for the longitudinal direction, index $\mathrm{T}$ is for the transverse direction, $\mathrm{A}$ is the amplitude and $\mathrm{O}$ is the circumference, $\lambda$ is the wavelength, $v$ is the frequency, $\mathrm{T}$ is the period, $\mathrm{c}$ is the light speed, $\gamma$ is the Lorentz factor.

Table 3. Doppler's Longitudinal Blueshift of the Super-Elastic WAVE

Doppler's Longitudinal Blueshift of the Super-Elastic WAVE

$$
\begin{aligned}
& \nu_{L}=\nu_{T}=\nu_{0}\left(1+\frac{v}{c}\right) \gamma=\nu_{0} \frac{\sqrt{1+\frac{v}{c}}}{\sqrt{1-\frac{v}{c}}} \\
& \lambda_{L}=\frac{\lambda_{0}}{1+\frac{v}{c}} \gamma^{-1}=\lambda_{0} \frac{\sqrt{1-\frac{v}{c}}}{\sqrt{1+\frac{v}{c}}} \\
& c_{L}=c \\
& T_{L}=T_{T}=T_{0} \gamma^{-1} \\
& A=\frac{\lambda_{0}}{2 \pi} \gamma \\
& O=2 \pi \frac{\lambda_{0}}{2 \pi} \gamma \\
& c_{T}={ }_{c} \gamma^{2}
\end{aligned}
$$

\section{Zwicky's Longitudinal Redshift of the Super-Elastic WAVE}

Table 4. Zwicky's Longitudinal Redshift of the Super-Elastic WAVE

$$
\begin{aligned}
& \text { Zwicky's Longitudinal Redshift of the Super-Elastic WAVE } \\
& \frac{m_{1}}{m_{0}}=\frac{\nu_{1}}{\nu_{0}}=\frac{\lambda_{0}}{\lambda_{1}}=e^{-H t}=\frac{1}{1+z} \\
& \lambda_{L}=\lambda_{0}(1+z) \\
& \nu_{L}=\nu_{T}=\frac{\nu_{0}}{1+z} \\
& c_{L}=c \\
& T_{L}=T_{T}=T_{0}(1+z) \\
& A=\frac{\lambda_{0}}{2 \pi} \frac{1}{1+z} \\
& O=2 \pi \frac{\lambda_{0}}{2 \pi} \frac{1}{1+z} \\
& c_{T}=\frac{c}{(1+z)^{2}}
\end{aligned}
$$

Tolman's Surface Brightness Test

$$
B=\frac{m_{C_{T}}^{2}}{T}=\frac{m_{0}}{(1+z)} \frac{c^{2}}{(1+z)^{2}} \frac{1}{T_{0}(1+z)}=\frac{m_{0} c^{2}}{T_{0}} \frac{1}{(1+z)^{4}}
$$


When the photon source is far away from the observer, the massive photon evaporates its mass with the evaporation rate given by the photon mass evaporation rate (Hubble constant) $\mathrm{H}=(2.2 \pm 0.1) * 10^{-18} \mathrm{~kg} \mathrm{~kg}^{-1} \mathrm{~s}^{-1}$. Zwicky's model of the Super-Elastic WAVE predicts the properties of that WAVE given in the Table 4. Many of those properties were already very well experimentally documented for the model of the expanding Universe. We propose to measure the amplitudes of those "tired" Zwicky's Super-Elastic WAVES.

In the Table 4: index $\mathrm{L}$ is for the longitudinal direction, index $\mathrm{T}$ is for the transverse direction, $\mathrm{A}$ is the amplitude and $\mathrm{O}$ is the circumference, $\mathrm{m}$ is the mass of the photon, $\lambda$ is the wavelength, $v$ is the frequency, $\mathrm{T}$ is the period, $\mathrm{c}$ is the light speed, $\mathrm{H}$ is the Hubble constant, $\mathrm{t}$ is time for the arrival of the photon from the source to the observer. B stands for the surface brightness in the Tolman's test.

\section{Lorentz' Acting Force of Redshifted Photons and Reacting Force of Fermions-Pushing of Fermions}

We have to follow the advice of Albert Einstein: "It is not good to introduce the concept of mass $\mathrm{M}=\gamma \mathrm{m}$ of a moving body for which no clear definition can be given". For details see L.B. Okun (1989) who made a great effort to remove this meme of the "relativistic mass" from our thinking.

H.A. Lorentz derived his famous formula for the longitudinal force $F_{L}=\gamma^{3} m_{L}$ and for the transverse force $F_{T}=$ $\gamma \mathrm{ma}_{\mathrm{T}}$ that were excellently confirmed by all experiments.

We propose that the occurrence of the Lorentz factor come from the microworld-from properties of redshifted photons pushing fermions with the acting force while fermions react against the action by their reaction.

Table 5 summarizes acting force of the redshifted photons and the reacting force of fermions. In that Table 5 index $\mathrm{L}$ describes the longitudinal motion, index $\mathrm{T}$ describes the transverse motion, $\mathrm{m}_{0}$ is the mass of photon, $\mathrm{c}_{\mathrm{T}}$ light velocity in the transverse direction, $\gamma$ is the Lorentz factor, $m_{e}$ is the fermion mass, $a_{L}$ is the acceleration in the longitudinal direction, $\mathrm{a}_{\mathrm{T}}$ the acceleration in the transverse direction.

Table 5. Acting force of redshifted photons in the longitudinal direction

Acting force of redshifted photons in the longitudinal direction

$\vec{F}_{L}^{R}=\frac{m_{0} c_{T}^{2}}{\lambda_{L}}=m_{0} c^{2} \gamma^{-2} \frac{\gamma^{-1}}{\lambda_{0}}=\frac{m_{0} c^{2}}{\lambda_{0}} \gamma^{-3}$

Reacting force of fermions in the longitudinal direction

$\stackrel{\leftarrow}{F}_{L}^{E}=\gamma^{3} m_{e} a_{L}$

Acting force of redshifted photons in the transverse direction

$\vec{F}_{T}^{R}=\frac{m_{0} c_{T}^{2}}{\lambda_{T}}=m_{0} c^{2} \gamma^{-2} \frac{\gamma}{\lambda_{0}}=\frac{m_{0} c^{2}}{\lambda_{0}} \gamma^{-1}$

Reacting force of fermions in the transverse direction

$\stackrel{\leftarrow}{F}_{T}^{E}=\gamma m_{e} a_{T}$

\section{Lorentz' Acting Force of Blueshifted Photons and Reacting Force of Fermions-Braking of Fermions}

We have to follow the advice of Albert Einstein: "It is not good to introduce the concept of mass $\mathrm{M}=\gamma \mathrm{m}$ of a moving body for which no clear definition can be given". For details see L.B. Okun (1989) who made a great effort to remove this meme of the "relativistic mass" from our thinking.

H.A. Lorentz derived his famous formula for the longitudinal force $F_{L}=\gamma^{3} \mathrm{ma}_{\mathrm{L}}$ and for the transverse force $\mathrm{F}_{\mathrm{T}}=$ $\gamma \mathrm{ma}_{\mathrm{T}}$ that were excellently confirmed by all experiments. In this experimental modification the fermions have being pushed by photons. What will be the situation for the braking of fermions by photons? We propose to study the braking of fermions in more details.

For this case we propose that the occurrence of the Lorentz factor come from the microworld-from properties of blueshifted photons braking fermions with the acting force while fermions react against this action by their reaction.

Table 6 summarizes acting force of the blueshifted photons and the reacting force of fermions. In that Table 6 index $\mathrm{L}$ describes the longitudinal motion, index $\mathrm{T}$ describes the transverse motion, $\mathrm{m}_{0}$ is the mass of photon, $\mathrm{c}_{\mathrm{T}}$ 
light velocity in the transverse direction, $\gamma$ is the Lorentz factor, $m_{e}$ is the fermion mass, $a_{L}$ is the acceleration in the longitudinal direction, $\mathrm{a}_{\mathrm{T}}$ the acceleration in the transverse direction.

Table 6. Acting force of blueshifted photons in the longitudinal direction

Acting force of blueshifted photons in the longitudinal direction

$\vec{F}_{L}^{B}=\frac{m_{0} c_{T}^{2}}{\lambda_{L}}=m_{0} c^{2} \gamma^{2} \frac{\gamma}{\lambda_{0}}=\frac{m_{0} c^{2}}{\lambda_{0}} \gamma^{3}$

Reacting force of fermions in the longitudinal direction

$\stackrel{\leftarrow}{F}_{L}^{E}=\gamma^{-3} m_{e} a_{L}$

Acting force of blueshifted photons in the transverse direction

$\vec{F}_{T}^{B}=\frac{m_{0} c_{T}^{2}}{\lambda_{T}}=m_{0} c^{2} \gamma^{2} \frac{\gamma^{-1}}{\lambda_{0}}=\frac{m_{0} c^{2}}{\lambda_{0}} \gamma$

Reacting force of fermions in the transverse direction

$\overleftarrow{\leftarrow}_{T}^{E}=\gamma^{-1} m_{e} a_{T}$

\section{9. (Superconducting) Magnetic Flux Quantum $\Theta_{0}$ of Photons}

We will model the photon with its mass $\mathrm{m}$ as the dyon rotating on the helical path (see Table 1 where we have postulated properties for that dyon). This is the full wave-Huygens-de Broglie wave. We will use "the old recipe" of Isaac Newton and will introduce the locus of centers of curvature of this full helical wave. It is known that the locus of centers of curvature of a cylindrical helix is a coaxial helix of equal pitch. This Newton-Bohm helical empty evolute will guide that photon on its helical path. The rotating dyon on the helical path with the Weber speed $\mathrm{c}_{\mathrm{W}}=\sqrt{ } 2 \mathrm{c}$ will create self-organized magnetic field in the direction of the central axis of that cylindrical helix. The helix angle of the cylindrical helix is $\alpha=45^{\circ}$. We have summarized some properties for that photon. (This was a great surprise to obtain the expression for the (superconducting) magnetic flux $\Theta_{0}$ for photons (April 072019 ) based on the Feynman Lectures, Vol II, Chapter 29).

Table 7. The expression of the (superconducting) magnetic flux quantum $\Theta_{0}$ for photons

Guiding force for the photon on the helical orbit

$F=\frac{m c_{W}^{2}}{\rho}=e c_{W} B \cos \alpha$

where $\rho$ is the radius of curvature of that cylindrical helix, B is magnetic flux density, $e=e / 2-(-e / 2)=e$.. electric charge of that rotating dyon on the cylindrical helix, $\alpha=45^{\circ}$ is the helix angle, $\mathrm{c}_{\mathrm{W}}$ is Weber's speed, $\mathrm{m}$ is the photon mass

$F=\frac{2 \pi m c^{2}}{\lambda}=\operatorname{ec} B$

Magnetic flux density B

$B=\frac{2 \pi m c}{\lambda e} \quad\left[W b m^{-2}\right]$

The (superconducting) magnetic flux quantum $\Theta_{0}$ for the surface $\mathrm{S}$ of the top of the cylinder

$\Theta_{0}=\frac{2 \pi m c}{\lambda e} S=\frac{2 \pi m c}{\lambda e} \pi\left(\frac{\lambda}{2 \pi}\right)^{2}=\frac{h}{2 e} \approx 2.067833848 \ldots{ }^{*} 10^{-15} \mathrm{~Wb}$

It was very unexpected result to get the expression of the (superconducting) magnetic flux quantum $\Theta_{0}$ for photons!!!

It was very unexpected result to get the expression of the (superconducting) magnetic flux quantum $\Theta_{0}$ for photons!!! It could completely change our approach for the study of high-temperature superconductors. In this case we should try to create complex of electron with the dyon (photon) in such a way that it will remains Table 
1 and the composite material. Temperature of the used matrix could increase the vibration of the matrix atoms and thus can destroy this valuable complex electron-dyon (photon). Based on the work of K.S. Tikhonov et al. (2018) we can derive the condition:

$$
\frac{3}{2} \frac{k_{B} T_{C}}{e} \geq \frac{h \nu}{2 e}
$$

where $\mathrm{k}_{\mathrm{B}}$ is the Boltzmann constant, $\mathrm{T}_{\mathrm{C}}$ is the critical temperature of the superconductor.

There are very well-known methods to specialists in this field how to work with micro-WAVES and infrared WAVES to stimulate and modify the superconductivity (e.g., A. Cavalleri in 2017, A. Saraiva et al. in 2017). We want to pass this model to experienced Readers of this Journal to evaluate the potential of this model. At this moment we see a possibility to go with photon wavelength close to the visible spectrum before the photoelectric effect will emit free electrons as it was documented by Philipp Lenard, Albert Einstein and Robert Andrews Millikan. Electrical voltage difference emf should fulfill this condition:

$$
\text { emf }=\frac{h}{2 e}\left(\nu_{\text {crit }}-\nu\right)
$$

where $v_{\text {crit }}$ is the frequency where the photoelectric effect will start to emit free electrons.

\section{Observation of the Visible Huygens-De Broglie Helical Orbit in the Plato's Cave}

We can experimentally observe the full wave only-the particle on the Huygens-de Broglie helical path from the Aristotelian World. The empty wave-Newton-Bohm guiding helical evolute cannot be experimentally detected, it is the "hidden" mathematical object in the Plato's Realm.

The result of the experiment with the full wave will depend on the position of the observer from which angle he will observe that particle. Observers in the Plato's Cave can see circle, sine, cosine, trochoid, cochleoid, and hyperbolic spiral. Therefore, we are confronted with the Rashomon effect: many observers-many results for the same event. There are known about fourteen different interpretations of Quantum Mechanics. We propose to name this Rashomon effect as "Many Interpretations of One World".

This concept brings a new insight to this topic. The probability of the finding the particle at a particular location in space is proportional to the square of the height of the corresponding helical wave at that point-Born rule. We might derive the Heisenberg relation as:

$$
\frac{\lambda}{2 \pi} \sin ^{2} \alpha m \sqrt{2} \quad c \cos \alpha=\frac{h}{4 \pi}
$$

where the helix angle $\alpha=45^{\circ}$.

There is the very well-known quotation of J.S. Bell: "No local hidden variable theory (in the Spirit of Einstein's ideas) can reproduce QM predictions for EPR correlations at all orientations of polarizers".

If we can prepare two particles on the entangled helical paths we might state the J.S. quotation: "Two particles on the entangled helical paths can reproduce QM predictions for EPR correlations at all orientations of polarizers."

\section{Conclusions}

1. We have combined knowledge of Old Masters, New Masters, and Dissidents for the formulation of the Super-Elastic WAVE as the model for Photon.

2. We propose to study amplitudes of those Super-Elastic WAVES in the Doppler's redshift experiments.

3. We propose to study amplitudes of those Super-Elastic WAVES in the Doppler's blueshift experiments.

4. We propose to study amplitudes of those Super-Elastic WAVES in the Zwicky's redshift experiments.

5. We propose to study reacting force of fermions during their braking by photons.

6. We propose to study the (superconducting) magnetic flux quantum $\Theta_{0}$ of photons in order to optimize the design of superconductors.

7. Nature might hide Her Beauty in plain sight protected by the mathematical camouflage.

8. Are there some more "hidden curves" in the Plato's Realm connected to the Photon Secrets? How to distinguish the real physical meaning written in those curves from fictious events if both are mathematically correct? How to 
work with the mathematical camouflage used by Nature to protect Her Secrets?

9. We want to pass this model into hands of Readers of this Journal better educated in Mathematics and Physics.

\section{Acknowledgments}

This work was supported by the JP\&FŠ Agency (Contract Number 25g/1963), by the VZ\&MŠ Agency (Contract Number 16000/1989) and by the GMS Agency (Contract Number 69110/1992). We were supported by the contract number $28101918 / 2018$. We have found the valuable support on the web site www.wolframalpha.com with the corrections of used formulae.

\section{Conflict of interests}

The authors declare that there is no conflict of interests regarding the publication of this paper.

\section{References}

Abbe, E. K. (1873). About a New Illumination Apparatus to the Microscope (pp. 469-480). Archiv für mikroskopische Anatomie, Verlag von Max Cohen \& Sons, Bonn.

Ashworth, R. A. (1998). Confirmation of Helical Travel of Light through Microwave Waveguide Analysis. Physics Essays, 11, 1-10. Retrieved from https://www.researchgate.net/publication/260829054 Confirmation_of_Helical_Travel_of_Light_through_Microwave_Waveguide_Analyses

Assis, A. K. T. (2003). On the First Electromagnetic Measurement of the Velocity of Light by Wilhelm Weber and Rudolf Kohlrausch. In F. Bevilacqua \& E. A. Giannetto (Eds.), Volta and the History of Electricity (pp. 267-286). Retrieved from https://www.ifi.unicamp.br/ assis/Weber-Kohlrausch(2003).pdf

Bardini, G., \& Gianella, G. M. (2016). A Historical Walk along the Idea of Curvature, from Newton to Gauss Passing from Euler. International Mathematical Forum, 11, 259-278. Retrieved from http://www.m-hikari.com/imf/imf-2016/5-8-2016/p/bardiniIMF5-8-2016.pdf

Bell, J. S. (1992). Six Possible Worlds of Quantum Mechanics. Foundations of Physics, 22, 1201-1215.

Blaschke, P. (2017). Pedal Coordinates, Dark Kepler and Other Forces Problems. Arxiv: 1704.00897v1.

Blåsjö, V. (2017). Transcendental Curves in the Leibnizian Calculus. Academic Press, Elsevier, ISBN: 978-0-12-813237-1.

Blåsjö, V. (2018). Mathematicians Versus Philosophers in Recent Work on Mathematical Beauty. Journal of Humanistic Mathematics, 8, 414-431. Retrieved from https://scholarship.claremont.edu/cgi/viewcontent.cgi? referer=https://www.google.com/\&httpsredir=1\&article=1380\&context $=\mathrm{jhm}$

Bohm, D. (1952). A Suggested Interpretation of the Quantum Theory in Terms of Hidden Variables. I. Physical Review, 85, 166-179.

Bohm, D. (1989). Quantum Theory. Dover Publications, ISBN-10: 9780486659695.

Böhm, J. (2016). Wonderful World of Pedal Curves. Retrieved from http://rfdz.ph-noe.ac.at/fileadmin/Mathematik_Uploads/ACDCA/TIME2016/Boehm_Pedals_.pdf

Born, M. (1926). Zur Quantenmechanik der Stoßvorgänge. Zeitschrift für Physik, 37.

Brackenridge, J. B. (1996). The Key to Newton's Dynamics: The Kepler Problem and the Principia. Berkeley: University of California Press. ISBN: 978-0520202177.

Bruneau, O. (2015). ICT and History of Mathematics: The Case of the Pedal Curves from 17th Century to 19th Century. Retrieved from https://hal.archives-ouvertes.fr/hal-01179909/document

Cavalleri, A. (2017). Photo-induced Superconductivity. Contemporary Physics.

Chashchina, O., Dudisheva, N., \& Silagadze, Z. K. (2019). Voigt Transformations in Retrospect: Missed Opportunities? Arxiv: 1609.08647v2.

Circular Polarization. Retrieved from https://en.wikipedia.org/wiki/Circular_polarization

Cohen, I. B. (1999). A Guide to Newton's Principia. The Principia: The Mathematical Principles of Natural Philosophy. Berkeley, CA, University California Press. ISBN: 978-0-520-08816-0.

Cohen, I. B., \& Smith, G. E. (Eds.) (2004). The Cambridge Companion to NEWTON. Cambridge University Press, Cambridge, ISBN 0-521-65696-6.

Cook, T. A. (1914). The Curves of Life. Constable and Company, London. 
Cylindrical Helix. Retrieved from http://demonstrations.wolfram.com/SineAndCosineHelix/

Cylindrical Helix. Retrieved from http://mathworld.wolfram.com/Helix.html

Cylindrical Helix. Retrieved from https://en.wikipedia.org/wiki/Helix

Cylindrical Helix. Retrieved from https://www.mathcurve.com/courbes3d.gb/helicecirculaire/helicecirculaire. shtml

Darrigol, O. (2012). A History of Optics. From Greek Antiquity to the Nineteenth Century. Oxford University Press. Oxford. ISBN-10: 0199644373.

De Broglie, L. (1927). Electrons et Photons: Rapport et Discussions du Cinquième Conseil de Physique tenu à Bruxelles du 24 au 29 Octobre 1927. Gauthier-Villars.

De Broglie, L. (1939). Matter and light: The New Physics (pp. 136-142). W.W: Norton CO., New York.

De Broglie-Bohm Theory. Retrieved from https://en.wikipedia.org/wiki/De_Broglie\%E2\%80\%93Bohm_theory

Dennis, D. (1995). Historical Perspectives for the Reform of Mathematics Curriculum: Geometric Curve Drawing Devices and their Role in the Transition to an Algebraic Description of Functions. Dissertation at Cornell University. Retrieved from http://www.quadrivium.info/mathhistory/CurveDrawingDevices.pdf

Dirac, P. (1931). Quantised Singularities in the Electromagnetic Field. Proc. Roy. Soc. (London) A, 133, 60.

Doppler, C. (1842). About the Colored Light of the Binary Stars and Some Other Stars of the Heavens. Proceedings of the Royal Bohemian Society of Sciences (Part V, Vol. 2). Prague.

Dürer, A. (1525). Underweysung der Messung, mit dem Zirckel und Richtscheyt, in Linien, Ebenen und gantzen corporen. (Dürer's Plan for the Cylindrical Helix in a Staircase). Retrieved from https://commons.wikimedia.org/wiki/Category:Underweysung_der_Messung

Dyon. Retrieved from https://en.wikipedia.org/wiki/Dyon

Einstein, A. (1905). Über einen die Erzeugung und Verwandlung des Lichtes betreffenden heuristischen Gesichtpunkt. Annalen der Physik, 17, 132-148.

Einstein, A. (1905). Zur Elektrodynamik bewegter Körper. Annalen der Physik, 322, 891-921.

Ernst, A., \& Hsu, J. P. (2001). First Proposal of the Universal Speed of Light by Voigt in 1887. Chin. J. Phys., $39,211-230$.

Euclid, L. (n. d.). Apollonius of Perga. On the Cochlias. Friedlein, 105, 1-6.

Evans, M. W. (1993). The Photon's Magnetic Flux Quantum ВП: The Magnetic Nature of Antisymmetric Light Scattering. The Photon Magnetic Field, 227-242.

Feynman, R. (1964). Motion of Charges in Electric and Magnetic Fields. Feynman Lectures (Vol II., Chapter 29). Retrieved from http://www.feynmanlectures.caltech.edu/II_29.html

Freire, O. (2003). Science and Exile: David Bohm, the Hot Times of the Cold War, and his Struggle for a New Interpretation of Quantum Mechanics. Retrieved from https://arxiv.org/ftp/physics/papers/0508/0508184.pdf

Freire, O. (2015). The Quantum Dissidents: Rebuilding the Foundations of Quantum Mechanics (1950-1990). With a Forword by S.S. Schweber. Springer, ISBN-10: 9783662446614.

Fried, M., \& Unguru, S. (2001). Apollonius of Perga's Conica: Text, Context, Subtext. Mnemosyne, Bibliotheca Classic. ISBN-10: 9004119779.

Gant, de F. (1995). Force and Geometry in Newton's Principia. Princeton University Press, Princeton. ISBN 0-691-03367-6.

Gell-Mann, M. (1964). A Schematic Model of Baryons and Mesons. Physics Letters, 8, 214-215.

Geminus of Rhodes: Cylindrical Helix. Retrieved from https://www-history.mcs.st-and.ac.uk/Biographies/Geminus.html

Guicciardini, N. (2003). Reading the Principia: The Debate on Newton's Mathematical Methods for Natural Philosophy from 1687 to 1736. Cambridge University Press, Cambridge. ISBN-10: 0521544033.

Hadot, P. (2008). The Veil of Isis: An Essay on the History of the Idea of Nature. Belknap Press. ISBN-10: 0674030494. 
Hall, A. R. (2015). Philosophers at War: The Quarrel Between Newton and Leibniz. Cambridge University Press. ISBN: 052152489X.

Hardy, L. (1992). On the Existence of Empty Waves in Quantum Theory. Physics Letters A, 167, 11-16.

Heat, T. L. (2015). Apollonius of Perga: Treatise on Conic Sections. Carruthers Press. ISBN-10: 1446021262.

Heisenberg, W. (1927). Über den anschaunlichen Inhalt der quantentheoretischen Kinematik und Mechanik. Zeitschrift für Physik, 43, 172-198.

Helical Electromagnetic Waves. (2011). Retrieved from http://blackholeformulas.com/files/EnM.html

Heras, R. (2014). Voigt's Transformations and the Beginning of the Relativistic Revolution. Arxiv: 1411.2559v1.

Heron, L. (1912). Heronis Alexandrini opera quae supersunt omnia.Translated into German by J.L. Heiberg.

History of Special Relativity. Retrieved from https://en.wikipedia.org/wiki/History_of_special_relativity

Huygens, Chr. (1678). Traité de la Lumière, Leyden, Van der Aa. (In Treatise on Light, London: McMillan, 1912).

Imaginary Time. Retrieved from https://en.wikipedia.org/wiki/Imaginary_time

$\begin{array}{llll}\text { Interpretations of } & \text { Quantum } & \text { Mechanics. } & \text { Retrieved }\end{array}$ https://en.wikipedia.org/wiki/Interpretations_of_quantum_mechanics

Köller, J. (2016). Fusspunktkurven und Gegenfusspunktkurven. Retrieved from http://www.mathematische-basteleien.de/fusspunktkurve.htm

Kvasz, L. (2012). Galileo, Descartes, and Newton-Founders of the Language of Physics. Acta Physica Slovaca, 62, 519-614. Retrieved from http://www.physics.sk/aps/pubs/2012/aps-12-06/aps-12-06.pdf

Laird, W. R., \& Roux, S. (Eds.) (2008). Mechanics and Natural Philosophy before the Scientific Revolution. Springer. ISBN 978-90-481-7491-1.

Lenard, P. (1902). Über die lichtelektrische Wirkung. Annalen der Physik, 313, 149-198.

Levitt, L. S. (1978). Is the Photon a Double Helix? Lettere al Nuovo Cimento, 21, 222-223.

Lewis, P. J. (2007). Empty Waves in Bohmian Quantum Mechanics. British Journal for the Philosophy of Science, 58, 787-803.

Lockwood, E. H. (1961). A Book of Curves. Cambridge University Press. Retrieved from http://www.aproged.pt/biblioteca/ABookofCurvesLockwood.pdf

Lorentz, H. A. (1904). Electromagnetic Phenomena in a System Moving with any Velocity Smaller than that of Light. Proceedings of the Royal Netherlands Academy of Arts and Sciences, 6, 809-831.

Mach, E. (1933). Die Mechanik in Ihrer Entwicklung, Wissenschaftliche Buchgesselschaft Darmstadt. ISBN 3-534-05873-9.

Mancosu, P., \& Arana, A. (2010). Descartes and the Cylindrical Helix. Historia Mathematica, 37, 403-427. Retrieved from https://www.sciencedirect.com/science/article/pii/S0315086009000949

Maor, E., \& Jost, E. (2014). Beautiful Geometry. Princeton University Press, Princeton. ISBN-13:978-0-691-15099-4.

Mass in special relativity. Retrieved from https://en.wikipedia.org/wiki/Mass_in_special_relativity

Millikan, R. (1914). A Direct Determination of "h". Physical Review, 4, 73-75.

Nauenberg, M. (1993). Newton's Early Computational Method for Dynamics. Retrieved from https://core.ac.uk/download/pdf/82476251.pdf

Nauenberg, M. (2018). Newton's Graphical Method for Central Force Orbits. Am. J. Phys., 86, 765-771.

Nauenberg, M. (2018). Visiting Newton's Atelier before the Principia, 1679-1684. Arxiv: 1805.06871v.

Negi et al. (2010). Generalization of Schwinger-Zwanziger Dyon to Quaternion. Arxiv: 1012.0279v1.

Newton, I. (1687). The Principia. Mathematical Principles of Natural Philosophy. Translated by I.B. Cohen and A. Whitman. University California Press, Berkeley. ISBN 978-0-520-08816-0.

Newton, I. (1704). Optics: Or, a treatise of the reflections, refractions, inflexions, and colors of light. Also two treatises of the species and magnitude of curvilinear figures. Palo Alto, Calif.: Octavo, ISBN 1-891788-04-3. 
Okun, B. L. (1989). The Concept of Mass (Mass, Energy, Relativity). Sov. Phys. Usp., 32, 629-638. Retrieved from http://www.itep.ru/science/doctors/okun/publishing_eng/em_5.pdf

Pedal Curves Properties. (2018). Retrieved from https://en.wikipedia.org/wiki/Pedal_curve

Pilot Wave Theory and Quantum Realism. (2016). Space Time, PBS Digital Studios. Retrieved from https://www.youtube.com/watch?v=RlXdsyctD50

Pilot Wave Theory. Retrieved from https://en.wikipedia.org/wiki/Pilot_wave_theory

Polchinski, J. (2003). Monopoles, Duality, and String Theory. Arxiv: hep-th/0304042v1

Ptolemy's Almagest (translated by G.J. Toomer, Foreword O. Gingerich). (1998). Princeton University Press, Princeton. ISBN-10: 0-691-00260-6.

Rashomon Effect. Retrieved from https://en.wikipedia.org/wiki/Rashomon_effect

Roveli, C. (2018). Physics Needs Philosophy. Philosophy Needs Physics. Found. Phys., 48, 481-491. Retrieved from https://arxiv.org/ftp/arxiv/papers/1805/1805.10602.pdf

Saglam et al. (2016). Quantum Flux Associated with a Photon on the Basis of the Heisenberg Uncertainty Relation. Results in Physics, 6, 215-216. Retrieved from https://www.sciencedirect.com/science/article/pii/S2211379716300122

Saraiva et al. (2017). Photonic Counterparts of Cooper Pairs. Arxiv: 1709.04520v2

Schwinger, J. (1969). A Magnetic Model of Matter. Science, 165, 757-761.

Seager, W. (2018). The Philosophical and Scientific Metaphysics of David Bohm. Entropy, 20, 493.

Shnir, Z. M. (2005). Magnetic Monopoles (Theoretical and Mathematical Physics). Springer, ISBN-10: 3540252770 .

Stávek, J. (2018). Kepler's Ellipse Generated by the Trigonometrically Organized Gravitons. Appl. Phys. Res., 10, 26-37.

Stávek, J. (2018). Kepler's Ellipse Observed from Newton's Evolute (1687), Horrebow's Circle (1717), Hamilton's Pedal Curve (1847), and Two Contrapedal Curves (28.10.2018). Appl. Phys. Res., 10, 90-101.

Stávek, J. (2019). Galileo's Parabola Observed from Pappus' Directrix, Apollonius'Pedal Curve (Line), Galileo's Empty Focus, Newton's Evolute, Leibniz's Subtangent and Subnormal, Ptolemy's Circle (Hodograph), and Dürer-Simon Parabola (16.03.2019). Appl. Phys. Res., 11, 56-72.

Stávek, J. (2019). Newton's Hyperbola Observed from Newton's Evolute (1687), Gudermann's Circle (1833), the Auxiliary Circle (Pedal Curve and Inversion Curve), the Lemniscate of Bernoulli (1694) (Pedal Curve and Inversion Curve) (09.01.2019). Appl. Phys. Res., 11, 65-78.

Stávek, J. (2019). Newton's Parabola Observed from Pappus' Directrix, Apollonius's Pedal Curve (Line), Newton's Evolute, Leibniz's Subtangent and Subnormal, Castillon's Cardioid, and Ptolemy's Circle (Hodograph) (09.02.2019). Appl. Phys. Res., 11, 183-195.

Strutz, Ch. (2001). Von Apollonius zur Himmelsmechanik. Retrieved from http://www.schulphysik.de/strutz/gravit2.pdf

Sugimoto, T. (2009). How to Present the Heart of Newton's Principia to the Layperson: A Primer on the Conic Sections without Apollonius of Perga. Symmetry: Culture and Science, 20, 113-144. Retrieved from http://www.is.kanagawa-u.ac.jp/overview/doc/Symmetry_Sugimoto_2009.pdf

Swetz, F. J., \& Katz, V. J. (2011). Mathematical Treasures-Albrecht Durer's Treatise on Mensuration. Covergence. Retrieved from https://www.maa.org/press/periodicals/convergence/mathematical-treasures-albrecht-durers-treatise-on-men suration

Tests of Special Relativity. Retrieved from https://en.wikipedia.org/wiki/Tests_of_special_relativity

Tikhonov, K. S., Skvortsov, M. A., \& Klapwijk, T. M. (2018). Superconductivity in the Presence of Microwaves: Full Phase Diagram. Physical Review B, 97, 184516.

Tired Light. Retrieved from https://en.wikipedia.org/wiki/Tired_light

Tolman Surface Brightness Test. Retrieved from https://en.wikipedia.org/wiki/Tolman_surface_brightness_test 
Tombe, F. D. (2019). The 1855 Weber-Kohlrausch Experiment (The Speed of Light). The General Science Journal. Retrieved from https://www.scribd.com/document/294114501/The-1855-Weber-Kohlrausch-Experiment-The-Speed-of-Lig ht

Voigt, W. (1887). Theorie des Licht für bewegte Medien. Göttinger Nachrichten, 8, 177-238.

Voigt, W. (1887). Über das Doppler'sche Prinzip. Gott. Nachr., 2, 41-51.

Watson, J. D. (2001). The Double Helix: A Personal Account of the Discovery of the Structure of DNA. Touchstone, ISBN-10: 9780743216302.

Weinstein, G. (2015). Einstein's Pathway to the Special Theory of Relativity. Cambridge, Cambridge Scholars Publishing.

Williams, G. (2019). Unravelling the Double Helix. The lost Heroes of DNA. Weidenfeld \& Nicholson, ISBN-10: $147460935 \mathrm{X}$.

Witten, E. (1979). Dyons of Charge e $\theta / 2 \pi$. Physics Letters B, 86, 283-287.

Wolfram, S. (2018). The History and Future of Special Functions. Retrieved from https://www.stephenwolfram.com/publications/history-future-special-functions/

Yates, R. C. (1974). Curves and their Properties. National Council of Teachers of Mathematics. ISBN: 10-087353039X.

Zweig, G. (1964). An SU(3) Model for Strong Interaction Symmetry and its Breaking. CERN Report No. 8182/TH.401.

Zwicky, F. (1933). Die Rotverschiebung von extragalaktischen Nebeln. Helv. Phys. Acta., 6, 110-127.

\section{Copyrights}

Copyright for this article is retained by the author(s), with first publication rights granted to the journal.

This is an open-access article distributed under the terms and conditions of the Creative Commons Attribution license (http://creativecommons.org/licenses/by/4.0/). 